\title{
Estimation of $\mathrm{R}=\mathrm{P}[\mathrm{Y}<\mathrm{X}]$ for Burr type $\mathrm{XII}$ distribution based on ranked set sampling
}

\author{
Amal S. Hassan, Assar, S. M, Yahya, M* \\ Modern Academy for Engineering \& Technology \\ *Corresponding author E-mail: Dr.Marwayahya@yahoo.com
}

Copyright $\odot 2014$ Amal S. Hassan et al. This is an open access article distributed under the Creative Commons Attribution License, which permits unrestricted use, distribution, and reproduction in any medium, provided the original work is properly cited.

\begin{abstract}
Ranked set sampling (RSS) is a statistical technique for data collection that generally leads to more efficient estimators than competitors based on simple random sampling (SRS). In the current paper, the estimation of $\mathrm{R}=\mathrm{P}[\mathrm{Y}<\mathrm{X}]$ when $\mathrm{Y}$ and $\mathrm{X}$ are two independent Burr type XII distribution with common known shape parameter $\mathrm{c}$ will be considered. Maximum likelihood estimator is proposed to estimate $\mathrm{R}$ based on ranked set sampling data. These estimators will be compared in terms of their biases, mean square errors and efficiencies with known estimators based on SRS data. It is shown that the estimators based on RSS are more efficient than the corresponding SRS. The results are illustrated using simulated data.
\end{abstract}

Keywords: Burr Type XII, Stress Strength Model, Ranked Set Sampling, Method of Maximum Likelihood.

\section{Introduction}

Burr [5] introduced twelve different forms of cumulative distribution functions which might be useful for fitting data, among those distributions Burr type XII, denoted by Burr $(c, b)$. The cumulative distribution function for Burr $(c, b)$ is given as

$F(x ; c, b)=1-\left(1+x^{c}\right)^{-b}, x>0, c>0, b>0$,

where $c$ and $b$ are shape parameters.

The corresponding probability density function $(p d f)$ is given by

$f(x ; c, b)=\mathrm{b} c x^{c-1}\left(1+x^{c}\right)^{-(b+1)}, x>0, c>0, b>0$.

The density (2) is unimodel at $\left.x=\left((c-1) /(b c+1)^{1 / 2}\right)\right)$.

RSS was proposed by McIntrye [2], he noted that in many situations, there is enough information available to enable sampling units to be ranked according to the variable of interest, but actually not measuring (quantifying) the units with respect to the variable of interest. The ranking may be done on the basis of visual inspection, or any prior information. The RSS method can be summarized as follows:

Step 1: Randomly select $n^{2}$ units from the target population.

Step 2: Allocate the $n^{2}$ selected units as randomly as possible into $n$ sets, each of size $n$.

$\left(\begin{array}{cccc}x_{11} & x_{12} & \ldots & x_{1 n} \\ \vdots & \vdots & \ldots & \vdots \\ x_{n 1} & x_{n 2} & \ldots & x_{n n}\end{array}\right)$

Step 3: Without taking any measurements, rank units within each raw based on a criterion chosen by the researcher. The result with one cycle can be represented as:

$\left(\begin{array}{cccc}x_{1(1)} & x_{1(2)} & \ldots & x_{1(n)} \\ \vdots & \vdots & \ldots & \vdots \\ x_{n(1)} & x_{n(2)} & \ldots & x_{n(n)}\end{array}\right)$

Step 4: Choose a sample for actual quantification by including the smallest ranked unit in the first set, the second smallest ranked unit in the second set, the process is continues in this way until the largest ranked unit is selected from the last set. 
Step 5: Repeat steps 1 through 4 for $r$ cycles to obtain a sample of size $n r$.

Takahasi and Wakimato [7] showed that RSS mean is an unbiased estimator for the population mean with smaller variance compared to the SRS mean. Dell and Clutter [11] showed that the mean of the RSS is an unbiased estimator of the population mean whether the ranking is perfect or not. Shen [13] explored the concept of RSS for the problem of estimation of a lognormal mean with a known coefficient of variation, and show that the use of RSS and its suitable modifications results is much improved estimators compared to the use of a SRS.

The estimation problem of $P(Y<X)$ has attracted the attention of many authors. This model first considered by Birnbaum [15]. The formal term stress-strength appeared in the title of Church and Harris [6]. The theoretical and practical results on the theory and applications of the stress-strength relationships in industrial and economic systems during the last decades are collected in Kotz et al. [9].

Awad and Charraf [1] provided a simulation study which compared three estimators for $R=P(Y<X)$ when $Y$ and $X$ are two independent but not identically distributed Burr random variables. These estimators are the minimum variance unbiased, maximum likelihood and Bayes. Panahi and Asadi [4] considered the estimation of $P(Y<X)$ when $X$ and $Y$ are independent identically random variables of Burr Type XII distribution with two parameters. They discussed the maximum likelihood estimator of $\mathrm{R}$ based on one simple iterative procedure assuming that the common parameter is known. They also, obtained the uniform minimum variance unbiased estimator (UMVUE) and Bayes estimator of R.

Recently, Sengupta and Mukhuti [10] considered the estimation problem of $R$ based on RSS, they compared the ranked set sampling with simple random sampling in terms of the variance of the unbiased estimator of $P(Y<X)$. They proved that the unbiased estimator based on RSS data has a smaller variance compared with the unbiased estimator based on SRS, even when the rankings of RSS are imperfect. Muttlak et al. [3] considered the problem of estimating $P(Y<X)$, where $X$ and $Y$ are independently exponentially distributed random variables with different scale parameters using ranked set sampling data. Abu Dieh et al. [12] introduced estimation of reliability $R$ based on data collected via RSS technique in case of normal distribution with concomitant variable. Dong et al. [14] considered the problem of estimating the reliability estimation $P(Y<X)$ for a system when strength $X$ and stress $\mathrm{Y}$ follow exponential distribution. They presented the best linear unbiased estimator and the modified maximum likelihood estimator based on ranked set sampling with unequal sampling. Hussian [8] discussed the problem of estimating R for generalized inverted exponential distribution based on RSS and SRS.

The main aim of this study is to focus on the estimation problem of $P(Y<X)$, where $X \sim \operatorname{Burr}(c, b)$ and $Y \sim \operatorname{Burr}(c, a)$ and they are independently distributed. The maximum likelihood estimator (MLE) and uniformly minimum variance unbiased estimator of R using SRS will be considered. MLE based on RSS will be derived. Simulation study is performed to compare different estimators.

The rest of the article is organized as follows. In Section 2, MLE and UMVUE of $R$ are discussed. In Section 3 , MLE of R based on RSS are obtained. Numerical study is presented in Section 4. Finally conclusions are presented in Section 5 .

\section{Methods of estimation of parameters in $\mathbf{R}$ based on SRS}

In this section, MLE and UMVUE of reliability $R$ will be considered. Let $X \sim \operatorname{Burr}(c, b)$ and $Y \sim \operatorname{Burr}(c, a)$ are two independent Burr type XII random variables, then it can be easily seen (see Awad and Charraf [1], and Panahi and Asadi [4]) that:

$R=P(Y<X)=\int_{0}^{\infty} \int_{0}^{x} b c x^{c-1}\left(1+x^{c}\right)^{-(b+1)} a c y^{c-1}\left(1+y^{c}\right)^{-(a+1)} d y d x=\frac{a}{a+b}=\frac{1}{1+\rho}$

where $\rho=\frac{b}{a}$.

To compute the point estimator of R, then firstly it must obtain the point estimator of unknown parameters $b$ and $a$. In the following subsection some point estimators of $R$ namely, $M L E$ and $U M V U E$ will be obtained.

\subsection{Maximum likelihood estimator}

To compute the MLE of $R$, the MLEs of $b$ and $a$ must be computed firstly. Let $X_{1}, \ldots, X_{p}$ be a SRS from Burr $(c, b)$ and $Y_{1}, \ldots, Y_{q}$ be a SRS from Burr $(c, a)$ therefore the log-likelihood function denoted by $l$ for the observed sample is given as follows:

$l=p \ln b+q \ln a+(p+q) \ln c+(c-1)\left[\sum_{i=1}^{p} \ln x_{i}+\sum_{j=1}^{q} \ln y_{j}\right]-(b+1) \sum_{i=1}^{p} \ln \left(1+x_{i}^{c}\right)-(a+1) \sum_{j=1}^{q} \ln \left(1+y_{j}^{c}\right)$.

The MLEs of $b$ and $a$ denoted by $\hat{b}_{M L E}, \hat{a}_{M L E}$ can be obtained as the solutions of:

$\frac{\partial l}{\partial b}=\frac{p}{\hat{b}_{M L E}}-\sum_{i=1}^{p} \ln \left(1+x_{i}^{c}\right)=0$,

$\frac{\partial l}{\partial a}=\frac{q}{\hat{a}_{M L E}}-\sum_{j=1}^{q} \ln \left(1+y_{j}^{c}\right)=0$.

From (5) and (6), the MLEs of $b$ and $a$, when c is known take the following forms:

$\hat{b}_{M L E}=\frac{p}{\sum_{i=1}^{p} \ln \left(1+x_{i}^{c}\right)^{\prime}}$

$\hat{a}_{M L E}=\frac{q}{\sum_{j=1}^{q} \ln \left(1+y_{j}^{c}\right)}$. 
Once the MLEs of $b$ and $a$ are obtained from (7) and (8) respectively, therefore, the MLE of R using SRS is given by $\widehat{R}_{M L E}=\frac{1}{1+\widehat{\rho}_{M L E}}$,

where $\hat{\rho}_{M L E}=\frac{\hat{b}_{M L E}}{\hat{a}_{M L E}}$. (see Awad and Charraf [1], and Panahi and Asadi [4]).

\subsection{Uniformly minimum variance unbiased estimator}

To obtain UMVUE of $\mathrm{R}$ let $\left(\sum_{i=1}^{p} \ln \left(1+x_{i}^{c}\right), \sum_{j=1}^{q} \ln \left(1+y_{j}^{c}\right)\right)$ is a jointly sufficient statistic for $(b, a)$, assuming that the common parameter $\mathrm{c}$ is known, then the UMVUE will be as follows:

$\hat{R}_{U M V U E}=\sum_{i=0}^{p-1}(-1)^{i} \frac{(q-1) !(p-1) !}{(q+i-1) !(p-i-1) !}\left(\frac{T_{2}}{T_{1}}\right)^{i}$ if $T_{2} \leq T_{1}$,

or

$\hat{R}_{U M V U E}=1-\sum_{i=0}^{q-1}(-1)^{i} \frac{(q-1) !(p-1) !}{(q-i-1) !(p+i-1) !}\left(\frac{T_{1}}{T_{2}}\right)^{i}$ if $T_{2}>T_{1}$,

where $T_{1}=\sum_{i=1}^{p} \ln \left(1+x_{i}^{c}\right)$ and $T_{2}=\sum_{j=1}^{q} \ln \left(1+y_{j}^{c}\right)$. (see Awad and Charraf [1], and Panahi and Asadi [4]).

\section{Estimating $\mathbf{R}=\mathbf{P}(\mathbf{Y}<X)$ using RSS}

This section deals with estimation of reliability $R$ using RSS, this approach to data collecting enables one to provide more structural to the collected sample items, and use this structure to develop efficient inferential procedures. MLE will be used to estimate the reliability $R=P(Y<X)$. Let $X_{(1: n) s}, X_{(2: n) s}, \ldots, X_{(n: n) s}$ for $i=1, \ldots, n$ and $s=1, \ldots, r$ is a RSS from Burr $(c, b)$, with sample size $p=n r$, where $n$ is the set size and $r$ is the number of cycles. To simplify the notation, denote $X_{(i: n) s}$ by $X_{i s}$, under the perfect judgment ranking, $X_{i s}$ follows the same distribution as $X_{(i: n) s}$, then for fixed $r, X_{i s}$ are independent with $p d f$ given by:

$f_{i}\left(x_{i s}\right)=\frac{n !}{(i-1) !(n-i) !}\left[F\left(x_{i s}\right)\right]^{i-1}\left[1-F\left(x_{i s}\right)\right]^{n-i} f\left(x_{i s}\right), \quad x_{i s}>0$.

Similarly, let $Y_{(1: m) s}, Y_{(2: m) s}, \ldots, y_{(m: m) s}$ for $j=1, \ldots, m$ and $s=1, \ldots, r$ is a $\operatorname{RSS}$ from $\operatorname{Burr}(c, a)$, with sample size $q=m r$, where $m$ is the set size. To simplify the notation, denote $Y_{(j: m) s}$ by $Y_{j s}$. Under the perfect judgment ranking, $Y_{j s}$ follows the same distribution as $Y_{(j: m) s}$, then for fixed $r, Y_{j s}$ are independent with $p d f$ given by:

$f_{j}\left(y_{j s}\right)=\frac{m !}{(j-1) !(m-j) !}\left[F\left(y_{j s}\right)\right]^{j-1}\left[1-F\left(y_{j s}\right)\right]^{m-j} f\left(y_{j s}\right), \quad y_{j s}>0$

Suppose $\left\{X_{i s}, i=1, \ldots, n ; s=1, \ldots, r\right\}$ denote the ranked set sample of size $p=n r$ from Burr $(c, b)$, where $n$ is the set size and $r$ is the number of cycles. Then the $p d f$ of $x_{i s}$ is given by:

$f_{i}\left(x_{i s}\right)=\frac{n !}{(i-1) !(n-i) !} b c x_{i s}^{c-1}\left(1+x_{i s}^{c}\right)^{-[b(n-i+1)+1]}\left(1-\left(1+x_{i s}^{c}\right)^{-b}\right)^{i-1}$.

By similar way, let $\left\{Y_{j s}, j=1, \ldots, m ; s=1, \ldots, r\right\}$ denote the ranked set sample of size $q=m r$ from Burr $(c, a)$. Then the $p d f$ of $y_{j s}$ is given by:

$f_{j}\left(y_{j s}\right)=\frac{m !}{(j-1) !(m-j) !} a c y_{j s}^{c-1}\left(1+y_{j s}^{c}\right)^{-[a(m-j+1)+1]}\left(1-\left(1+y_{j s}^{c}\right)^{-a}\right)^{j-1}$,

The likelihood function is given by:

$$
\begin{aligned}
L(c, b, a) & =\prod_{s=1}^{r}\left[\prod_{i=1}^{n} f_{i}\left(x_{i s}\right) \prod_{j=1}^{m} f_{j}\left(y_{j s}\right)\right] \\
& =\prod_{s=1}^{r}\left[\prod_{i=1}^{n} \frac{n !}{(i-1) !(n-i) !} b c x_{i s}^{c-1}\left(1+x_{i s}^{c}\right)^{-[b(n-i+1)+1]}\left(1-\left(1+x_{i s}^{c}\right)^{-b}\right)^{i-1} \prod_{j=1}^{m} \frac{m !}{(j-1) !(m-j) !} a c y_{j s}^{c-1}\right. \\
& \left.\times\left(1+y_{j s}^{c}\right)^{-[a(m-j+1)+1]}\left(1-\left(1+y_{j s}^{c}\right)^{-a}\right)^{j-1}\right]
\end{aligned}
$$

The log-likelihood function (16) denoted by $l$ will be as follows:

$l=\ln \xi+p \ln b+q \ln a+(c-1) \sum_{s=1}^{r}\left(\sum_{i=1}^{n} \ln x_{i s}+\sum_{j=1}^{m} \ln y_{j s}\right)-\sum_{s=1}^{r}\left[\sum_{i=1}^{n}[b(n-i+1)+1] \ln \left(1+x_{i s}{ }^{c}\right)\right.$

$\left.+\sum_{j=1}^{m}[a(m-j+1)+1] \ln \left(1+y_{j s}^{c}\right)\right]+\sum_{s=1}^{r}\left[\sum_{i=1}^{n}(i-1) \ln \left(1-\left(1+x_{i s}{ }^{c}\right)^{-b}\right)+\sum_{j=1}^{m}(j-1)\right.$

$\left.\times \ln \left(1-\left(1+y_{j s}{ }^{c}\right)^{-a}\right)\right]$

where $\xi$ is a constant. The first partial derivatives of log-likelihood function with respect to $b$ and $a$ are given by:

$\frac{\partial l}{\partial b}=\frac{p}{\tilde{b}_{M L E}}-\sum_{s=1}^{r}\left[\sum_{i=1}^{n}(n-i+1) \ln \left(1+x_{i s}^{c}\right)-\sum_{i=1}^{n}(i-1) \frac{\ln \left(1+x_{i s}^{c}\right)}{\left(1+x_{i s}^{c}\right)^{\tilde{b}_{M L E}}-1}\right]=0$,

$\frac{\partial l}{\partial a}=\frac{q}{\tilde{a}_{M L E}}-\sum_{s=1}^{r}\left[\sum_{j=1}^{m}(m-j+1) \ln \left(1+y_{j s}^{c}\right)-\sum_{j=1}^{m}(j-1) \frac{\ln \left(1+y_{j s}^{c}\right)}{\left(1+y_{j s}^{c}\right)^{\tilde{a}_{M L E}}}\right]=0$.

Clearly, it is not easy to obtain a closed form solution to this system of Equations (18) and (19). Therefore, an iterative technique must be applied to solve these equations numerically to obtain an estimates of $b$ and $a$. Let the MLEs of $b$ 
and $a$ denoted by $\tilde{b}_{M L E}$ and $\tilde{a}_{M L E}$ respectively. The MLE estimator $\tilde{R}_{M L E}$ of $R$ using RSS is obtained by substituting $\tilde{b}_{M L E}$ and $\tilde{a}_{M L E}$ in Equation (3). Then $\tilde{R}_{M L E}$ will be obtained as follows:

$\tilde{R}_{M L E}=\frac{1}{1+\widetilde{\rho}_{M L E}}$,

where $\tilde{\rho}_{M L E}=\frac{\tilde{b}_{M L E}}{\tilde{a}_{M L E}}$.

\section{Numerical illustration}

In this study, the problem of estimating $\mathrm{P}(\mathrm{Y}<X)$ has been addressed for Burr type XII distribution based on SRS and RSS. Simulation study is carried out to compare the estimators of R, $\widetilde{R}_{M L E}$ based on RSS to the estimators based on SRS data $\widehat{R}_{M L E}$ and $\widehat{R}_{U M V U E}$. Performance of estimators is evaluated through their biases, mean square errors (MSEs) and efficiencies. The simulation procedures are described through the following steps:

Step (1): Generate 1000 simple random samples of $x_{1}, x_{2}, \ldots, x_{p}$ and $y_{1}, y_{2}, \ldots, y_{q}$ from Burr type XII distribution with sample sizes $\{(n, m)=(30,30),(40,40),(50,50),(60,60),(30,40),(30,50)(30,60),(40,50),(40,60),(50,60)$, $,(40,30),(50,30)(50,40),(60,30),(60,40),(60,50)\}$.

Step (2): Generate 1000 simple random samples of $x_{11}, \ldots, x_{n r}$ and $y_{11}, \ldots, y_{m r}$ from Burr XII distribution with set sizes $n=3,4,5,6, m=3,4,5,6$ and number of cycles $r=10$.

Step (3): Without loss of generality the common parameter c will be assumed to be equal one in all the experiments. The ratio $\rho$ is selected as follows $\{0.1,0.5,1,2$ and 6$\}$.

Step (4): The biases, MSEs and efficiencies of estimators, namely $\left\{\tilde{R}_{M L E}, \hat{R}_{U M V U E}\right.$ and $\left.\hat{R}_{M L E}\right\}$ based on RSS and SRS are obtained. The efficiencies of the estimators with respect to $\hat{R}_{M L E}$ will be defined as follows eff $(1)=\frac{\hat{R}_{M L E}}{\hat{R}_{U M V U E}} \quad$ and $\operatorname{eff}(2)=\frac{\hat{R}_{M L E}}{\tilde{R}_{M L E}}$.

The simulation results are reported in Tables 1 to 3 and represented in Figures 1 to 5, the following conclusions can be observed:

1) The biases of all estimators $\tilde{R}_{M L E}, \hat{R}_{U M V U E}$ and $\hat{R}_{M L E}$ are very small.

2) In all cases, MSEs of $\hat{R}_{U M V U E}$ and $\hat{R}_{M L E}$ based on SRS data are greater than MSE of $\tilde{R}_{M L E}$ based on RSS data. (For example see Figure (1)).

3) In almost all cases, MSEs decrease as the set size increase. (For example see Figure (1)).

4) MSEs of all estimators $\widetilde{R}_{M L E}, \hat{R}_{U M V U E}$ and $\widehat{R}_{M L E}$ increase as the value of $\rho$ increases up to $\rho=1$ and then MSEs decrease as the value of $\rho$ increases. (For example see Figure (2)).

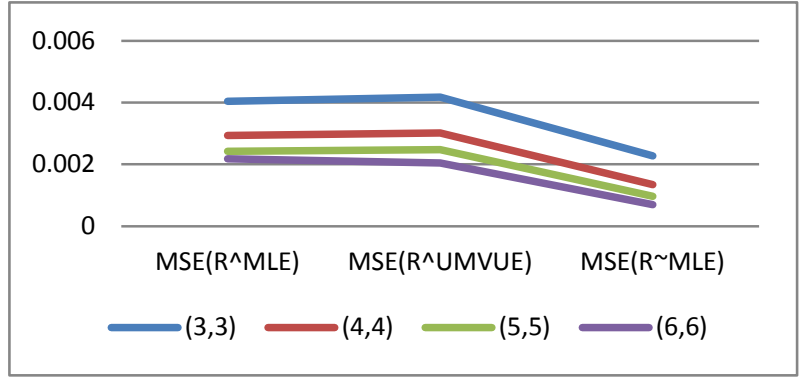

Fig. 1: MSEs for estimators $\widetilde{R}_{M L E}, \widehat{R}_{U M V U E}$ and $\widehat{R}_{M L E}$ for, $\rho=1$

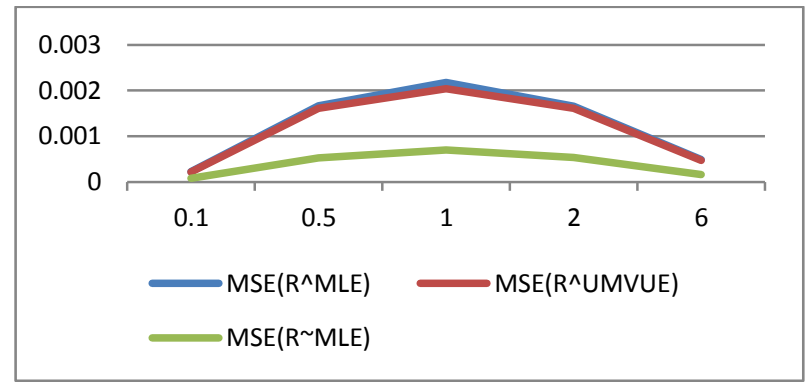

Fig. 2: MSEs for estimators $\tilde{R}_{M L E}, \hat{R}_{U M V U E}$ and $\hat{R}_{M L E}$ for, $(n, m)=(6,6)$ and different values of $\rho$

5) From Figure (2), it is observed that, the MSEs of all estimators have the smallest value when the value of $\rho=0.1$, while the MSEs of all estimators have the largest value when the value of $\rho=1$.

6) Efficiencies of $R$ based on RSS data with respect to SRS data are greater than one in all cases.

7) In case of $n=m$, the efficiency of $R$ based on RSS data with respect to SRS data is increasing as $n$ and $m$ are increasing for the same value of $\rho$. (For example see Figure (3)).

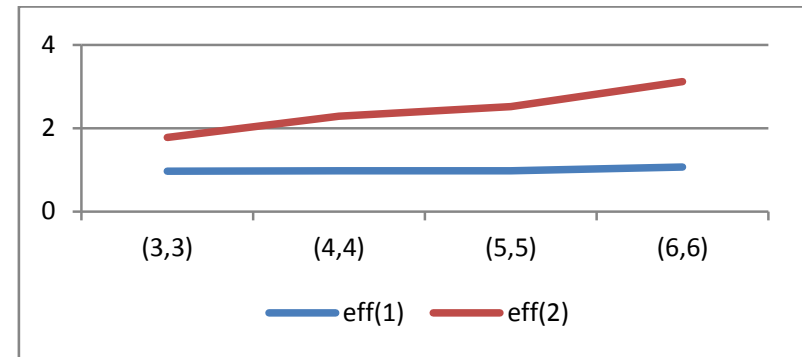

Fig. 3: Efficiencies for estimators $\widetilde{R}_{M L E}, \hat{R}_{U M V U E}$ with respect to $\hat{R}_{M L E}$ for $\rho=1$ 
8) In case of $n<m$ and if $n$ remains fixed $(n=3)$, the efficiency of $R$ based on RSS data with respect to SRS data is increasing as $m$ increases for the same value of $\rho$. (For example see Figure (4)).

9) In case of $n>m$ and if $n$ remains fixed $(n=6)$, the efficiency of $R$ based on RSS data with respect to SRS data is increasing as $m$ increases for the same value of $\rho$. (For example see Figure (5)).

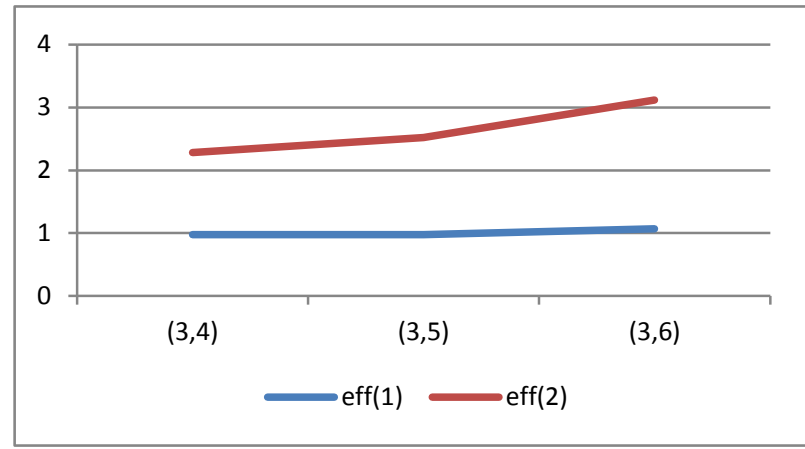

Fig. 4: The efficiencies of estimators $\tilde{R}_{M L E}, \hat{R}_{U M V U E}$ with respect to $\hat{R}_{M L E}$ when $n<m$ and $\rho=1$.

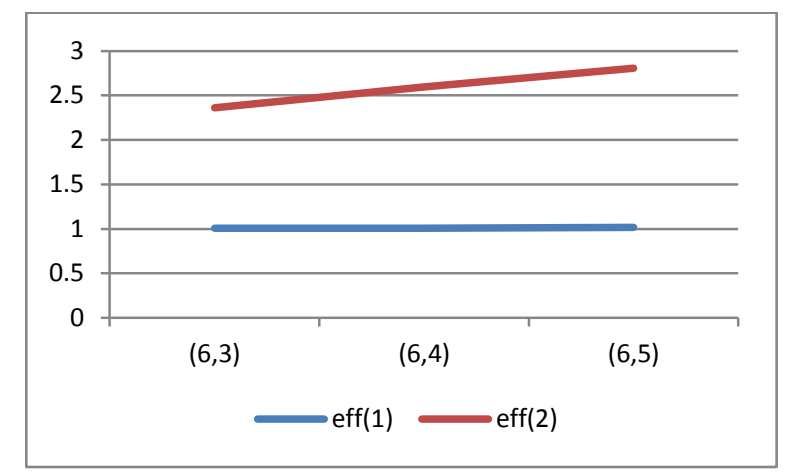

Fig. 5: The efficiencies of estimators $\tilde{R}_{M L E}, \hat{R}_{U M V U E}$ with respect to $\hat{R}_{M L E}$ when $n>m$ and $\rho=1$.

10) In almost all cases of equal set size, i.e., $(n=m)$, the efficiency of $\hat{R}_{U M V U E}$ with respect to $\hat{R}_{M L E}$ is greater than one at the values of $\rho=0.1$ and 6 , otherwise it is less than one .

11) In case of $(n \neq m)$, the efficiency of $\hat{R}_{U M V U E}$ with respect to $\hat{R}_{M L E}$ is greater than one in almost all cases.

12) $\tilde{R}_{M L E}$ based on RSS data is more efficient than $\hat{R}_{M L E}$ based on SRS in all cases.

13) $\tilde{R}_{M L E}$ based on RSS data is more efficient than $\hat{R}_{U M V U E}$ based on SRS in all cases.

\section{Conclusions}

This article deals with the estimation problem of $P(Y<X)$ when $X$ and $Y$ are assumed to follow Burr type XII distribution with parameters $(c, b)$ and $(c, a)$ respectively. In case of SRS and RSS, the performance of the estimators $\widetilde{R}_{M L E}, \hat{R}_{U M V U E}$ and $\hat{R}_{M L E}$ is investigated using the biases, MSEs and efficiencies.

From the simulation study, it is observed that, MSEs of all estimates decrease as the sample size increases in almost all cases. Based on RSS data $\tilde{R}_{M L E}$ has the smallest MSEs compared with the other methods of estimation $\hat{R}_{M L E}$ and $\hat{R}_{U M V U E}$ based on SRS data. In all cases the efficiency of estimating $R$ based on RSS data is greater than the efficiency of estimating $R$ based on SRS data. This study revealed that the estimators based on RSS approach are more efficient than the corresponding SRS.

Table 1: Biases, MSEs and Efficiencies for the Estimators $\widehat{\mathrm{R}}_{\mathrm{MLE}}, \widehat{\mathrm{R}}_{\mathrm{UMVUE}}$ and $\widetilde{\mathrm{R}}_{\mathrm{MLE}}$ Based on SRS and RSS when $n=m$.

\begin{tabular}{|c|c|c|c|c|c|c|c|c|c|c|c|}
\hline$\rho$ & $\mathrm{n}$ & $\mathrm{m}$ & $(p, q)$ & $\operatorname{Bias}\left(\widehat{\mathrm{R}}_{\mathrm{MLE}}\right)$ & $\operatorname{Bias}\left(\widehat{\mathrm{R}}_{\text {UMvUE }}\right)$ & $\operatorname{Bias}\left(\widetilde{\mathrm{R}}_{\mathrm{MLE}}\right)$ & $\operatorname{MSE}\left(\widehat{R}_{M L E}\right)$ & $\begin{array}{c}\text { MSE } \\
\left(\widehat{\mathrm{R}}_{\text {UMVUE }}\right)\end{array}$ & $\operatorname{MSE}\left(\widetilde{R}_{\mathrm{MLE}}\right)$ & $\operatorname{eff}(1)$ & $\operatorname{eff}(2)$ \\
\hline \multirow{4}{*}{0.1} & 3 & 3 & $(30,30)$ & -0.00294 & -0.00064 & -0.00109 & 0.00050 & 0.00047 & 0.00026 & 1.043 & 1.912 \\
\hline & 4 & 4 & $(40,40)$ & -0.00229 & 0.00016 & -0.00093 & 0.00035 & 0.00031 & 0.00015 & 1.098 & 2.303 \\
\hline & 5 & 5 & $(50,50)$ & -0.00048 & -0.00087 & -0.00074 & 0.00027 & 0.00026 & 0.00010 & 1.080 & 2.536 \\
\hline & 6 & 6 & $(60,60)$ & -0.00143 & 0.00048 & -0.00010 & 0.00023 & 0.00022 & 0.00008 & 1.022 & 2.913 \\
\hline \multirow{4}{*}{0.5} & 3 & 3 & $(30,30)$ & -0.00418 & -0.00418 & -0.00090 & 0.00328 & 0.00334 & 0.00181 & 0.981 & 1.806 \\
\hline & 4 & 4 & $(40,40)$ & -0.00348 & -0.00165 & -0.00131 & 0.00237 & 0.00240 & 0.00107 & 0.986 & 2.211 \\
\hline & 5 & 5 & $(50,50)$ & 0.00081 & -0.00081 & -0.00117 & 0.00191 & 0.00194 & 0.00076 & 0.984 & 2.506 \\
\hline & 6 & 6 & $(60,60)$ & -0.00116 & 0.00126 & -0.00133 & 0.00167 & 0.00161 & 0.00053 & 1.468 & 3.113 \\
\hline \multirow{4}{*}{1} & 3 & 3 & $(30,30)$ & -0.00197 & -0.00200 & 0.00050 & 0.00404 & 0.00418 & 0.00228 & 0.966 & 1.772 \\
\hline & 4 & 4 & $(40,40)$ & -0.00194 & -0.00256 & -0.00058 & 0.00293 & 0.00301 & 0.00134 & 0.973 & 2.282 \\
\hline & 5 & 5 & $(50,50)$ & 0.00253 & 0.00230 & -0.00067 & 0.00242 & 0.00247 & 0.00096 & 0.977 & 2.519 \\
\hline & 6 & 6 & $(60,60)$ & 0.00027 & 0.00137 & -0.000003 & 0.00218 & 0.00204 & 0.00070 & 1.069 & 3.114 \\
\hline \multirow{4}{*}{2} & 3 & 3 & $(30,30)$ & 0.00064 & -0.00182 & 0.00180 & 0.00321 & 0.00329 & 0.00182 & 0.975 & 1.764 \\
\hline & 4 & 4 & $(40,40)$ & 0.000009 & 0.00184 & 0.00028 & 0.00232 & 0.00236 & 0.00106 & 0.981 & 2.177 \\
\hline & 5 & 5 & $(50,50)$ & 0.00370 & 0.00224 & -0.00284 & 0.00195 & 0.00197 & 0.00076 & 0.989 & 2.559 \\
\hline & 6 & 6 & $(60,60)$ & -0.00014 & 0.00118 & 0.00011 & 0.00166 & 0.00161 & 0.00054 & 1.028 & 3.076 \\
\hline \multirow{4}{*}{6} & 3 & 3 & $(30,30)$ & 0.00190 & -0.00102 & 0.00186 & 0.00102 & 0.00101 & 0.00057 & 1.015 & 1.787 \\
\hline & 4 & 4 & $(40,40)$ & 0.00111 & -0.00108 & 0.00066 & 0.00072 & 0.00071 & 0.00032 & 1.008 & 2.201 \\
\hline & 5 & 5 & $(50,50)$ & 0.00298 & 0.00121 & 0.00034 & 0.00062 & 0.00060 & 0.00023 & 1.021 & 2.640 \\
\hline & 6 & 6 & $(60,60)$ & 0.00147 & -0.00139 & 0.00047 & 0.00049 & 0.00047 & 0.00016 & 1.042 & 3.096 \\
\hline
\end{tabular}


Table 2: Biases, MSEs and Efficiencies for the Estimators $\widehat{\mathrm{R}}_{\mathrm{MLE}}, \widehat{\mathrm{R}}_{\mathrm{UMVUE}}$ and $\widetilde{\mathrm{R}}_{\mathrm{MLE}}$ Based on SRS and RSS when $n<m$.

\begin{tabular}{|c|c|c|c|c|c|c|c|c|c|c|c|}
\hline$\rho$ & $\mathrm{n}$ & $\mathrm{m}$ & $(p, q)$ & $\begin{array}{c}\text { Bias } \\
\left(\widehat{\mathrm{R}}_{\mathrm{MLE}}\right)\end{array}$ & $\operatorname{Bias}\left(\widehat{\mathrm{R}}_{\text {UMVUE }}\right)$ & $\operatorname{Bias}\left(\widetilde{\mathrm{R}}_{\mathrm{MLE}}\right)$ & $\begin{array}{c}\text { MSE } \\
\left(\widehat{R}_{M L E}\right)\end{array}$ & $\begin{array}{c}\text { MSE } \\
\left(\widehat{\mathrm{R}}_{\text {UMVUE }}\right)\end{array}$ & $\operatorname{MSE}\left(\widetilde{R}_{M L E}\right)$ & $\operatorname{eff}(1)$ & $\operatorname{eff}(2)$ \\
\hline \multirow{6}{*}{0.1} & 3 & 4 & $(30,40)$ & -0.00291 & -0.00054 & -0.00121 & 0.00041 & 0.00038 & 0.00023 & 1.054 & 1.724 \\
\hline & 3 & 5 & $(30,50)$ & -0.00277 & -0.00020 & -0.00102 & 0.00038 & 0.00034 & 0.00018 & 1.092 & 2.108 \\
\hline & 3 & 6 & $(30,60)$ & -0.00140 & 0.00051 & -0.00094 & 0.00037 & 0.00032 & 0.00017 & 1.137 & 2.146 \\
\hline & 4 & 5 & $(40,50)$ & -0.00148 & 0.00064 & -0.00091 & 0.00033 & 0.00029 & 0.00013 & 1.132 & 2.572 \\
\hline & 4 & 6 & $(40,60)$ & -0.00188 & 0.00077 & -0.00045 & 0.00031 & 0.00026 & 0.00011 & 1.174 & 2.686 \\
\hline & 5 & 6 & $(50,60)$ & -0.00077 & 0.00061 & -0.00103 & 0.00025 & 0.00024 & 0.00009 & 1.018 & 2.783 \\
\hline \multirow{6}{*}{0.5} & 3 & 4 & $(30,40)$ & -0.00476 & -0.00171 & -0.00142 & 0.00269 & 0.00272 & 0.00164 & 0.987 & 1.637 \\
\hline & 3 & 5 & $(30,50)$ & -0.00425 & -0.00081 & -0.00351 & 0.00244 & 0.00246 & 0.00192 & 0.989 & 1.895 \\
\hline & 3 & 6 & $(30,60)$ & -0.00092 & -0.00045 & -0.00119 & 0.00253 & 0.00246 & 0.00122 & 1.025 & 2.078 \\
\hline & 4 & 5 & $(40,50)$ & 0.00146 & 0.00088 & -0.00145 & 0.00226 & 0.00231 & 0.00091 & 0.979 & 2.486 \\
\hline & 4 & 6 & $(40,60)$ & -0.00264 & -0.00021 & -0.00031 & 0.00215 & 0.00213 & 0.00082 & 1.006 & 2.603 \\
\hline & 5 & 6 & $(50,60)$ & -0.00151 & 0.00159 & -0.00074 & 0.00182 & 0.00177 & 0.00066 & 1.023 & 2.748 \\
\hline \multirow{6}{*}{1} & 3 & 4 & $(30,40)$ & -0.00312 & -0.00212 & -0.00021 & 0.00330 & 0.00339 & 0.00206 & 0.971 & 1.601 \\
\hline & 3 & 5 & $(30,50)$ & -0.00274 & -0.00111 & -0.00288 & 0.00302 & 0.00310 & 0.00160 & 0.974 & 1.889 \\
\hline & 3 & 6 & $(30,60)$ & 0.00108 & -0.00056 & -0.00031 & 0.00318 & 0.00309 & 0.00153 & 1.028 & 2.069 \\
\hline & 4 & 5 & $(40,50)$ & 0.00025 & 0.00088 & -0.00086 & 0.00283 & 0.00288 & 0.00114 & 0.980 & 2.468 \\
\hline & 4 & 6 & $(40,60)$ & -0.00117 & 0.00200 & 0.00034 & 0.00269 & 0.00245 & 0.00104 & 1.097 & 2.590 \\
\hline & 5 & 6 & $(50,60)$ & 0.00104 & 0.00176 & -0.00161 & 0.00231 & 0.00226 & 0.00073 & 1.022 & 3.146 \\
\hline \multirow{6}{*}{2} & 3 & 4 & $(30,40)$ & -0.00082 & -0.00207 & 0.00103 & 0.00259 & 0.00266 & 0.00164 & 0.974 & 1.582 \\
\hline & 3 & 5 & $(30,50)$ & -0.00064 & -0.00116 & -0.00161 & 0.00239 & 0.00245 & 0.00125 & 0.975 & 1.907 \\
\hline & 3 & 6 & $(30,60)$ & 0.00286 & -0.00054 & 0.00063 & 0.00256 & 0.00243 & 0.00122 & 1.052 & 2.091 \\
\hline & 4 & 5 & $(40,50)$ & 0.00190 & 0.00080 & -0.00009 & 0.00224 & 0.00227 & 0.00094 & 0.983 & 2.479 \\
\hline & 4 & 6 & $(40,60)$ & 0.00056 & 0.00167 & 0.00092 & 0.00215 & 0.00195 & 0.00082 & 1.099 & 2.613 \\
\hline & 5 & 6 & $(50,60)$ & 0.00230 & 0.00153 & -0.00100 & 0.00185 & 0.00179 & 0.00057 & 1.030 & 3.206 \\
\hline \multirow{6}{*}{6} & 3 & 4 & $(30,40)$ & 0.00078 & -0.00126 & 0.00135 & 0.00080 & 0.00080 & 0.00051 & 0.997 & 1.579 \\
\hline & 3 & 5 & $(30,50)$ & 0.00078 & -0.00073 & -0.00029 & 0.00075 & 0.00074 & 0.00038 & 1.071 & 1.960 \\
\hline & 3 & 6 & $(30,60)$ & 0.00281 & -0.00032 & 0.00093 & 0.00082 & 0.00074 & 0.00038 & 1.081 & 2.157 \\
\hline & 4 & 5 & $(40,50)$ & 0.00212 & 0.00045 & 0.00037 & 0.00070 & 0.00069 & 0.00027 & 1.153 & 2.529 \\
\hline & 4 & 6 & $(40,60)$ & 0.00134 & 0.00086 & 0.00090 & 0.00068 & 0.00060 & 0.00025 & 1.326 & 2.688 \\
\hline & 5 & 6 & $(50,60)$ & 0.00209 & 0.00082 & 0.00122 & 0.00050 & 0.00055 & 0.00017 & 1.454 & 2.829 \\
\hline
\end{tabular}

Table 3: Biases, MSEs and Efficiencies for Estimators $\widehat{\mathrm{R}}_{\mathrm{MLE}}, \widehat{\mathrm{R}}_{\mathrm{UMVUE}}$ and $\widetilde{\mathrm{R}}_{\mathrm{MLE}}$ Based on SRS and RSS when $n>m$.

\begin{tabular}{|c|c|c|c|c|c|c|c|c|c|c|c|}
\hline$\rho$ & $\mathrm{n}$ & $\mathrm{m}$ & $(p, q)$ & $\begin{array}{c}\text { Bias } \\
\left(\widehat{\mathrm{R}}_{\text {MLE }}\right)\end{array}$ & $\operatorname{Bias}\left(\widehat{\mathrm{R}}_{\text {UMvUE }}\right)$ & $\operatorname{Bias}\left(\widetilde{\mathrm{R}}_{\mathrm{MLE}}\right)$ & $\begin{array}{c}\mathrm{MSE} \\
\left(\widehat{\mathrm{R}}_{\mathrm{MLE}}\right)\end{array}$ & $\begin{array}{c}\text { MSE } \\
\left(\widehat{\mathrm{R}}_{\text {UMVUE }}\right)\end{array}$ & $\operatorname{MSE}\left(\widetilde{R}_{M L E}\right)$ & $\operatorname{eff}(1)$ & $\operatorname{eff}(2)$ \\
\hline \multirow{6}{*}{0.1} & 4 & 3 & $(40,30)$ & -0.00222 & 0.00059 & -0.00044 & 0.00041 & 0.00044 & 0.00020 & 0.904 & 2.041 \\
\hline & 5 & 3 & $(50,30)$ & -0.00150 & -0.00013 & -0.00059 & 0.00038 & 0.00035 & 0.00018 & 1.074 & 2.116 \\
\hline & 5 & 4 & $(50,40)$ & -0.00082 & 0.00067 & -0.00096 & 0.00030 & 0.00027 & 0.00013 & 0.998 & 2.251 \\
\hline & 6 & 3 & $(60,30)$ & -0.00030 & 0.00043 & -0.00018 & 0.00034 & 0.00032 & 0.00015 & 1.043 & 2.202 \\
\hline & 6 & 4 & $(60,40)$ & -0.00085 & 0.00003 & 0.00018 & 0.00028 & 0.00029 & 0.00011 & 1.013 & 2.424 \\
\hline & 6 & 5 & $(60,50)$ & -0.00103 & 0.00019 & -0.00078 & 0.00025 & 0.00027 & 0.00008 & 1.107 & 2.899 \\
\hline \multirow{6}{*}{0.5} & 4 & 3 & $(40,30)$ & -0.00282 & 0.00042 & 0.00037 & 0.00280 & 0.00300 & 0.00144 & 0.933 & 1.950 \\
\hline & 5 & 3 & $(50,30)$ & 0.00036 & -0.00017 & -0.00231 & 0.00271 & 0.00025 & 0.00128 & 1.066 & 2.107 \\
\hline & 5 & 4 & $(50,40)$ & 0.00014 & 0.00021 & -0.00154 & 0.00214 & 0.00215 & 0.00094 & 1.022 & 2.264 \\
\hline & 6 & 3 & $(60,30)$ & -0.00162 & 0.00020 & 0.00069 & 0.00258 & 0.00215 & 0.00109 & 1.033 & 2.353 \\
\hline & 6 & 4 & $(60,40)$ & -0.00235 & -0.00040 & -0.00002 & 0.00217 & 0.00212 & 0.00084 & 1.080 & 2.592 \\
\hline & 6 & 5 & $(60,50)$ & -0.00157 & 000163 & -0.00013 & 0.00177 & 0.00173 & 0.00067 & 1.039 & 2.617 \\
\hline \multirow{6}{*}{1} & 4 & 3 & $(40,30)$ & -0.00082 & -0.00106 & 0.00164 & 0.00352 & 0.00399 & 0.00182 & 0.876 & 1.926 \\
\hline & 5 & 3 & $(50,30)$ & 0.00257 & -0.00105 & 0.00122 & 0.00338 & 0.00360 & 0.00155 & 0.943 & 2.180 \\
\hline & 5 & 4 & $(50,40)$ & 0.00169 & 0.00378 & -0.00094 & 0.00271 & 0.00282 & 0.00118 & 0.960 & 2.303 \\
\hline & 6 & 3 & $(60,30)$ & 0.00118 & 0.00031 & 0.00161 & 0.00356 & 0.00306 & 0.00151 & 1.005 & 2.359 \\
\hline & 6 & 4 & $(60,40)$ & 0.00111 & 0.00003 & 0.00002 & 0.00280 & 0.00277 & 0.00106 & 1.011 & 2.595 \\
\hline & 6 & 5 & $(60,50)$ & -0.00097 & 0.00023 & -0.00002 & 0.00229 & 0.00226 & 0.00081 & 1.016 & 2.808 \\
\hline \multirow{6}{*}{2} & 4 & 3 & $(40,30)$ & 0.00137 & 0.00110 & 0.00255 & 0.00284 & 0.00298 & 0.00147 & 0.951 & 1.932 \\
\hline & 5 & 3 & $(50,30)$ & 0.00231 & 0.00356 & 0.00054 & 0.00289 & 0.00278 & 0.00127 & 1.038 & 2.280 \\
\hline & 5 & 4 & $(50,40)$ & 0.00337 & 0.00037 & -0.00014 & 0.00202 & 0.00206 & 0.00092 & 0.979 & 2.370 \\
\hline & 6 & 3 & $(60,30)$ & 0.00653 & -0.00218 & 0.00120 & 0.00264 & 0.00245 & 0.00107 & 1.075 & 2.456 \\
\hline & 6 & 4 & $(60,40)$ & 0.00141 & -0.00333 & -0.00051 & 0.00208 & 0.00199 & 0.00082 & 1.052 & 2.533 \\
\hline & 6 & 5 & $(60,50)$ & 0.00298 & -0.00099 & 0.00191 & 0.00181 & 0.00178 & 0.00066 & 1.012 & 2.742 \\
\hline \multirow{6}{*}{6} & 4 & 3 & $(40,30)$ & 0.00213 & 0.00021 & 0.00211 & 0.00091 & 0.00083 & 0.00046 & 0.962 & 1.977 \\
\hline & 5 & 3 & $(50,30)$ & 0.00355 & 0.00192 & 0.00134 & 0.00088 & 0.00093 & 0.00041 & 0.946 & 2.143 \\
\hline & 5 & 4 & $(50,40)$ & 0.00292 & 0.00028 & 0.00036 & 0.00070 & 0.00076 & 0.00028 & 1.043 & 2.488 \\
\hline & 6 & 3 & $(60,30)$ & 0.00243 & 0.00059 & 0.00184 & 0.00080 & 0.00076 & 0.00035 & 1.041 & 2.269 \\
\hline & 6 & 4 & $(60,40)$ & 0.00248 & 000079 & 0.00186 & 0.00062 & 0.00064 & 0.00026 & 1.241 & 2.381 \\
\hline & 6 & 5 & $(60,50)$ & 0.00273 & 0.00071 & 0.00055 & 0.00056 & 0.00054 & 0.000194 & 1.464 & 2.931 \\
\hline
\end{tabular}

\section{References}

[1] A. M. Awad and M. K. Charraf. Estimation of $P(Y<X)$ in Burr Case: A comparative study, Communications in Statistics - Simulation and Computation, 15 (1986), 389-403.

[2] G.A. McIntyre. A method for unbiased selective sampling, using ranked sets. Australian Journal of Agricultural Research, 3 (1952), $385-390$. 
[3] H.A. Muttlak, W.A. Abu-Dayyah, M.F. Saleh and E.Al-Sawi. Estimating $P(Y<X)$ using ranked set sampling in case of the exponential distribution. Communications in Statistics: Theory and Methods, 39 (2010), 1855-1868.

[4] H. Panahi and S. Asadi. Estimation of $R=P(Y<X)$ for two-parameter Burr type XII distribution. World Academy of Science, Engineering and Technology, 72 (2010), 465-470.

[5] I.W. Burr. Cumulative frequency functions. Annals of Mathematical Statistics, 13 (1942), 215-232.

[6] J. D. Church and B. Harris. The estimation of reliability from stress strength relationships. Technometrics, 12 (1970), 49-54.

[7] K.Takahasi and K.Wakimoto. On unbiased estimates of the population mean based on the sample stratified by means of ordering. Annals of the Institute of Statistical Mathematics, 20 (1968), 1-31.

[8] M. A. Hussian. Estimation of stress-strength model for generalized inverted exponential distribution using ranked set sampling. International Journal of Advances in Engineering \& Technology. 6(6) (2014), 2354-2362.

[9] S.Kotz, Y.Lumelskii, and M. Pensky. The stress-strength model and its generalizations: Theory and applications. World Scientific Publishing, (2003), ISBN 981-238-057-4.

[10] S.Sengupta and S. Mukhuti. Unbiased estimation of $P(X>Y)$ using ranked set sample data. Statistics, 42 (2008), 223-230.

[11] T.R. Dell and J. L. Clutter. Ranked set sampling theory with order statistics background. Biometrics, 28 (1972), 545-553.

[12] W. Abu-Dieh, A.Dorvlo and O. AL-Saidy. Estimation of reliability in case of bivariate normal distribution using ranked set sampling with concomitant variable. International Journal of the Computer, the internet and management, 19 (2011), 16.1-16.4.

[13] W. H. Shen. On the estimation of lognormal mean using a ranked set sample. The Indian Journal of Statistics, 56 (1994), 323-333.

[14] X. Dong, L. Zhang and F.Li. Estimation of reliability for exponential distributions using ranked set sampling with unequal samples. Quality Technology and Quantitative Management, 10 (2013), 319-328.

[15] Z.W. Birnbaum. On the use of Mann-Whitney statistics. Proceedings of the Third Berkeley Symposium in mathematical Statistics and Probability, 1 (1956), 13-17. 